\title{
Closed reduction and percutaneus Kirschner wire fixation for the treatment of dislocated calcaneal fractures: surgical technique, complications, clinical and radiological results after 2-10 years
}

\author{
Tim Alexander Walde $\cdot$ B. Sauer $\cdot$ J. Degreif $\cdot$ \\ H.-J. Walde
}

Received: 17 December 2006 / Published online: 29 February 2008

(C) The Author(s) 2008

\begin{abstract}
Introduction To reduce complications, a minimally invasive technique for the treatment of dislocated intraarticular fractures of the calcaneus was used. Therefore previously described closed reduction and internal fixation techniques were combined and modified.

Materials and methods Sixty-seven out of 92 calcaneal fractures could be retrospectively evaluated with an average follow-up time of 5.7 years (minimum 2-10 years follow-up). For radiographic evaluation, plain radiographs and CT scans were obtained. The Zwipp score was used for clinical evaluation. Sanders type II, III and IV fractures were diagnosed.

Results Length of surgery averaged 61 min (range 20-175 $\mathrm{min}$ ). The incidence of subtalar arthritis was correlated to the severity of fracture. Böhler's angle was restored in $70.1 \%$ (47 of 67) of the cases. On the last follow-up evaluation the average Zwipp score was 130 points (range 48-186 points). The majority (77.7\%) of patients were content with their treatment result. The rate of significant complications was $6.5 \%$.
\end{abstract}

\section{T. A. Walde $(\bowtie)$}

Department of Traumatology, Plastic and Reconstructive Surgery, University of Goettingen, Robert-Koch Straße 40,

37099 Göttingen, Germany

e-mail: timwalde@hotmail.com

B. Sauer · H.-J. Walde

Department of Traumatology, Hand and Reconstructive Surgery, Nordwest-Krankenhaus Sanderbusch, Hauptstraße, 26452 Sande, Germany

\section{J. Degreif}

Department of Traumatology and Orthopaedic Surgery, Städtische Kliniken Esslingen, Hirschlandstraße 97,

73730 Esslingen, Germany
Discussion Compared to open techniques the presented minimally invasive technique showed comparable results with a low rate of serious complications and is a viable alternative for the treatment of intraarticular, dislocated calcaneal fractures.

Keywords Dislocated calcaneal fracture . Minimal-invasive

\section{Introduction}

A fracture of the calcaneus allowed to heal in improper anatomical position leads to static and dynamic malfunctions of the whole foot with consequent limited load bearing capacity and walking ability $[4,29]$. The associated pain leads to a significant impairment in quality of life. The goal of therapy for calcaneal fractures is the elimination of pain and restoration of walking ability for patients with normal foot shape and the ability to wear normal footwear.

At present, there are multiple operative procedures for the treatment of calcaneal fractures. The procedures can be differentiated by approach, implant type and whether the treatment is one- or two-stages. Recently, open procedures using internal fixation have been favored for surgical therapy of the calcaneus $[2,5,9,15,17,23,24,26]$. A possible complication of an open procedure is the disturbance of wound healing with skin and soft tissue necrosis [2] and the possibility of cutaneous flaps [5, 8, 9, 15, 17]. In addition to posttraumatic arthritis in the lower ankle and adjoining joints, there are reports of osteitis of the calcaneus [2, 5, 17]. Advanced osteitis of the calcaneus can require a calcanectomy [15] or amputation of the lower leg [9, 17].

In an effort to reduce the complications that can occur with an open procedure, we have combined and modified 
previously described closed reduction and internal fixation techniques $[14,27,28]$ to create a minimally invasive technique.

The aim of this study was to evaluate the clinical and radiographic results of our minimally invasive surgical treatment of intraarticular fractures of the calcaneus at 2-10 years postoperatively. We then compared our results to the results of both open and minimally invasive surgical techniques found in the literature.

\section{Material and methods}

\section{Patients}

A total of 88 patients with 92 closed, dislocated and intraarticular fractures of the calcaneus were consecutively treated with a minimally-invasive technique developed at our institution by modifying and combining the procedures of Westhues [27], Poigenfürst and Buch [14], and Wondrák [28]. All the surgeries were performed without the use of bone graft. The average age at time of calcaneal fracture was 46.1 years (range 18-82 years) and most patients were male (71.6\%). The cause of fracture was a fall from varying heights in 75 $(85.2 \%)$ of cases and a motor vehicle accident in 13 (14.8\%) of cases. From this group of patients, 63 (71.6\%) patients with 67 fractures were available for retrospective examination with an average follow-up time of 5.7 years (range 2-10 years). Twenty-five patients were unavailable for followup examination; 12 patients who satisfied with the result of the surgery did not wish to participate in the study due to age or unwillingness to travel to the hospital, 7 patients were at an unknown address and 6 patients deceased.

\section{Examination}

All the medical records, radiographs, pre- and post-operative computed tomography scans were available for the entire study group. Traumatic soft tissue damage was determined using the classification method of Tscherne and Oestern [25]. In addition to radiographic evaluation using the calcaneus lateral, calcaneus axial and Broden views at $20^{\circ}$ and $40^{\circ}$ [3], all the patients obtained a bilateral calcaneal preoperative CT scan for fracture classification and surgical planning. Fractures were evaluated by the classification scale of Sanders et al. [17, 18]. The duration of surgery, as well as the time the X-ray image intensifier used, were noted as operative data. For postoperative evaluation of the reduction, radiographs of the calcaneus in lateral, axial, Broden $20^{\circ}$ and $40^{\circ}$ [3] views were obtained. At the last follow-up evaluation, a CT scan of both calcanei was obtained to examine the geometry of the calcaneus and to evaluate arthritic changes in the lower ankle joint.
For a reconstruction of the calcaneal posterior facet to be considered satisfactory a joint deviation of $\leq 2 \mathrm{~mm}$ had to be seen radiographically on the postoperative radiographs. For the evaluation of the clinical results, the Zwipp score was used [30].

\section{Statistical analysis}

For statistical analysis the Fisher's exact test and the Chisquare test were used.

Perioperative treatment and surgical technique

After a calcaneal fracture was diagnosed, the lower leg was evaluated. If the soft tissue was in good condition, primary surgery performed. In cases of severe swelling with potential soft tissue damage NSAIDs, local cryotherapy and active movement exercises determined the course of therapy.

Once the soft tissue was in good condition, surgery was performed. The patient was placed prone on the traction table under general anaesthesia or spinal anaesthesia without arrest of blood supply. After application of the calcaneus wire for traction in the dorso-cranial plane, the varus or valgus malaligment of the back foot was corrected with the wire positioned orthogonally to the longitudinal axis of the calcaneus. To achieve correct placement, the surgeon pulled at the traction bow with the knee bent along the longitudinal axis of the calcaneus. The subsequent traction in the plantar plane was performed with the leg stretched. Once placed in the proper orientation, the traction bow was connected to the retaining jig of the traction table (traction of $20 \mathrm{~kg}$ ). The surgeon then held the heel of the patient with both hands and applied compression both medially and laterally with his thenar muscle to reduce the main medial and lateral fragments.

With the use of the X-ray image intensifier, the restoration of Böhler's angle and the reduction of the posterior facet were verified. A Steinmann pin was put with the pointed end into a universal chuck and the other end into the dorso-lateral calcaneus beneath the posterior facet with a stab incision (Fig. 1a). For reduction of the posterior facet, the traction wire was used as a hypomochlion (Fig. 1b). The anatomical restoration of the posterior facet was verified with use of the Brodén radiographic views $\left(20^{\circ}\right.$ and $\left.40^{\circ}\right)$. The reduction of the calcaneus was verified by examining the lateral radiographic view (Fig. 2a). The fragments were fixed with percutaneus Kirschner wires, which were arranged conically into the talus and/or cuboid (Fig. 2b). All of the main fragments to be reduced were held in place by the Kirschner wires and the arch of foot was held until the bone healed [28]. The wires were bent above the skin level, the wire traction removed and the stab incision closed with sutures.

Postoperatively, a dorsal lower leg splint was applied for immobilization and the lower portion of the operative side 
Fig. 1 The Steinmann pin is placed into the dorso-lateral calcaneus above the traction wire (left side). For the reduction of the posterior facet, the traction wire is used as a hypomochlion (right side). The hematome is drained though the stab incision

Fig. 2 Intraoperative lateral radiograph of a dislocated calcaneal fracture before reduction (left side). After reduction the fragments are fixed with percutaneus Kirschner wires (right side)
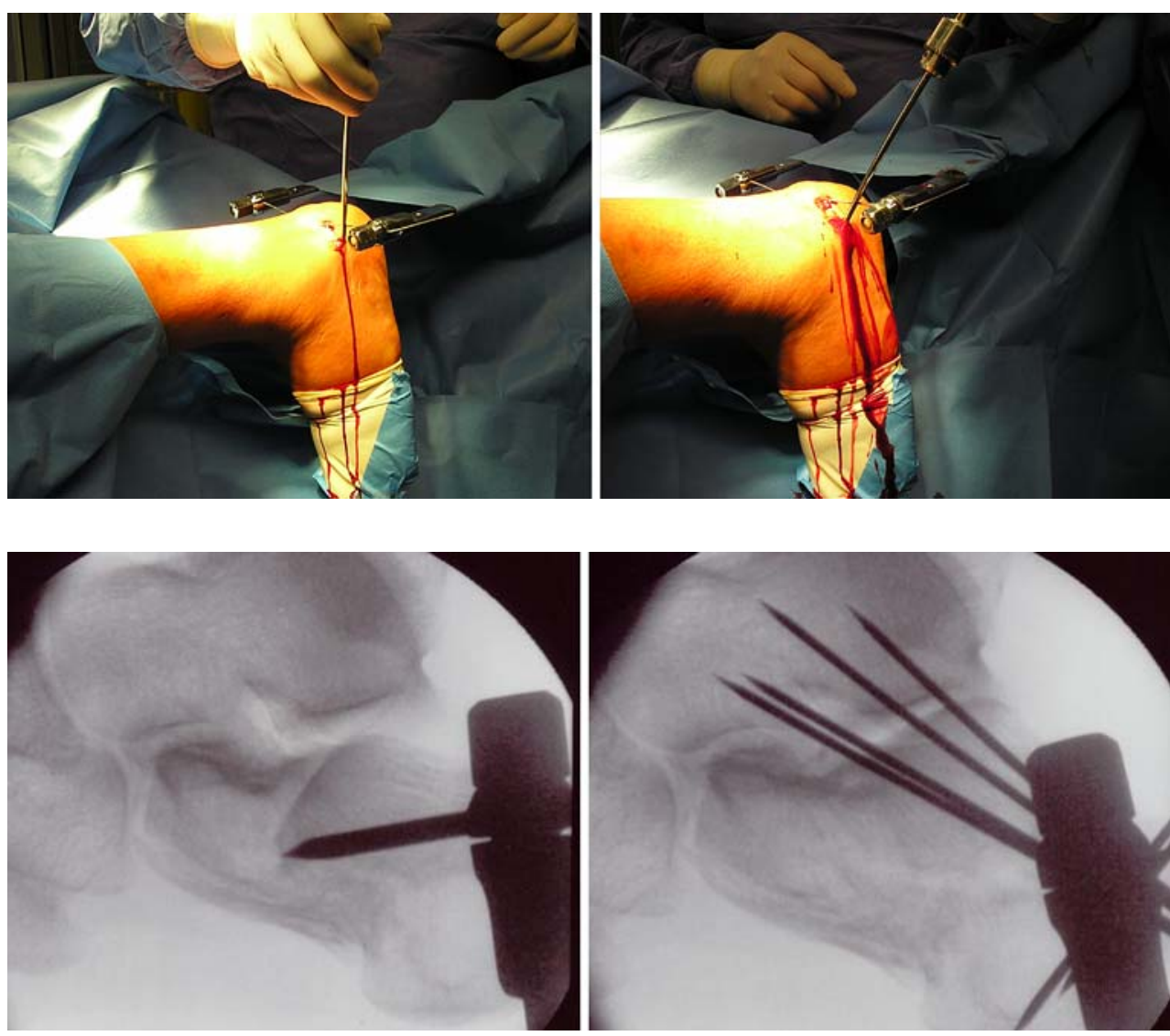

was elevated. On the first postoperative day, the patient was mobilized on crutches with no weight bearing on the operative side. Depending on pain levels, the patient began with active dorsal extension and plantar flexion in the upper ankle joint. The radiographic controls, (upper ankle joint anterior and lateral, calcaneus axial Brodén views [25]) were performed immediately postoperative, and again 2 and 8 weeks postoperative. After the fracture was healed, the Kirschner wires were removed. Partial weight bearing began after the eighth postoperative week at $20 \mathrm{~kg}$ and was increased up to full weight bearing by the twelfth postoperative week (Fig. 3).

\section{Results}

Fifty-five of the $88(62.5 \%)$ patients suffered exclusively from calcanal fractures. Thirty-three of the $88(37.5 \%)$ patients had multiple injuries. Twenty of the $33(61 \%)$ patients with multiple injuries had local co-injuries such as fractures of the upper ankle joint, tarsus and front-foot. In 83 of the 92 calcaneal fractures, the soft tissue injury was graded as $1^{\circ}$ or $2^{\circ}$; in 9 fractures, the soft tissue injury was graded as $3^{\circ}$. In eight cases, including three patients with multiple injuries, surgery was performed immediately on the day of the trauma.
Length of surgery averaged $61 \mathrm{~min}$ (range 20-175 min) and screening time averaged $115 \mathrm{~s}$ (range 20-454 s). To obtain proper retention of the fracture, between 4 and 7 Kirschner wires were used. The Kirschner wires were removed with or without local anaesthesia at an average of 10 weeks (range 7-15 weeks) as an outpatient procedure. Full weight bearing was achieved at an average of 15 weeks postoperative.

\section{Sanders classification}

Sanders type II, III and IV fractures were diagnosed (Table 1). All the patients had a joint deviation of at least $2 \mathrm{~mm}$. Patients with type I non-dislocated fractures were treated conservatively and were therefore not included in this study. Reconstruction of the posterior facet was verified radiographically in 13 of $15(86.7 \%)$ type II fractures, in 47 of $52(90.4 \%)$ type III fractures and in 16 of $25(64 \%)$ type IV fractures immediately postoperatively (Table 1).

The Zwipp score of 67 patients at the last follow-up evaluation

The Zwipp score of the 67 patients available for follow-up averaged $130 / 200$ points (range $48-186$ points) which is considered a good result [30]. Regardless of fracture type, 

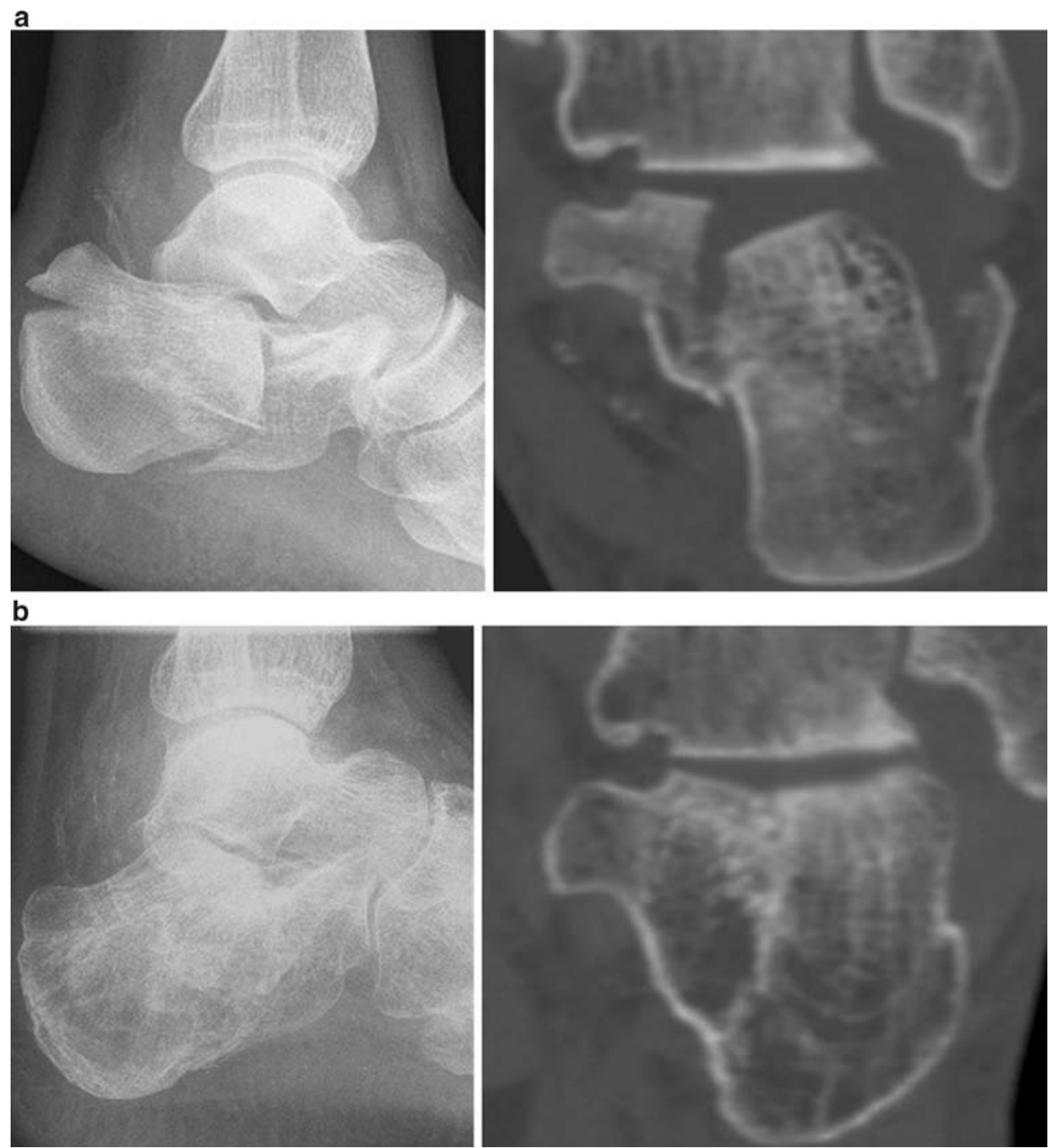

Fig. 3 Clinical example. a Preoperative lateral radiograph of an intraarticular, displaced calcaneal fracture (left side). The preoperative CT scan shows a Sanders Type II AC fracture on the coronal reconstruction (right side). b Lateral radiograph taken 2.5 years postoperatively.

Table 1 Patients with immediate postoperative reconstruction of the posterior facet by Sanders fracture classification

\begin{tabular}{lll}
\hline $\begin{array}{l}\text { Sanders fracture classification } \\
(N=92)\end{array}$ & $\begin{array}{l}\text { Radiological reconstruction } \\
\text { of posterior facet achieved }\end{array}$ \\
\hline Type II & $15(16.3 \%)$ & $13(86.7 \%)$ \\
Type III & $52(56.5 \%)$ & $47(90.4 \%)$ \\
Type IV & $25(27.2 \%)$ & $16(64.0 \%)$ \\
\hline
\end{tabular}

$41(61.2 \%)$ of the 67 patients had a very good or good result, $24(35.8 \%)$ patients had a satisfactory result and 2 (3\%) patients had a bad result. The worst clinical results occurred with type IV fractures (Table 2). Thirty $(44.8 \%)$ patients considered their treatment outcome very good or
The patient had a Zwipp score of 166 points (left side). Anatomical reconstruction of the posterior facet was achieved (right side). This coronal CT image shows the anatomical reconstruction of the posterior facet with no joint deviation

good, $28(41.8 \%)$ as satisfactory and sufficient and 9 $(13.4 \%)$ as insufficient.

Thirty-seven $(55.3 \%)$ of the 67 patients had no pain while full weight bearing or could walk at least $4 \mathrm{~h}$ without pain at last follow-up evaluation. Nine (13.4\%) patients had constant pain. Thirty-three $(49.3 \%)$ patients had a range of motion in the upper ankle joint identical to that of the nonaffected side. Thirty-four $(50.7 \%)$ patients had a restricted range of motion of up to $15^{\circ}$ and more than half $(58.2 \%)$ of the patients had achieved more than $75 \%$ of their total range of motion in the lower ankle joint. Forty-three $(64.2 \%)$ of 67 patients were able to wear normal shoes while $5(7.4 \%)$ used shoes with an unroll aid. Nineteen $(28.3 \%)$ patients had obtained orthopaedic shoes on their own at last follow-up. 
Table 2 Clinical and radiological results at last follow-up evaluation by Sanders fracture classification

\begin{tabular}{lcccc}
\hline $\begin{array}{l}\text { Sanders fracture } \\
\text { classification }\end{array}$ & $\begin{array}{l}\text { Very good } \\
\text { or good result }\end{array}$ & $\begin{array}{l}\text { Arthritis in lower } \\
\text { ankle joint }\end{array}$ & $\begin{array}{l}\text { Normal Böhler } \\
\text { angle achieved }\end{array}$ \\
\hline Overall & 67 & $41(61.2 \%)$ & $33(49.3 \%)$ & $47(70.1 \%)$ \\
Type II & $6(8.9 \%)$ & $4(66.7)$ & $1(16.7 \%)$ & $5(83.3 \%)$ \\
Type III & $39(58.2 \%)$ & $29(74.4 \%)$ & $12(30.8 \%)$ & $31(79.5)$ \\
Type IV & $22(32.8 \%)$ & $8(36.4 \%)$ & $20(90.9 \%)$ & $11(50.0 \%)$ \\
\hline
\end{tabular}

Two $(3 \%)$ of 67 patients had arthritis of the upper ankle joint at last follow-up. Independent of fracture type 33 (50.2\%) patients had arthritic changes in the lower ankle joint and $14(20.89 \%)$ cases had arthritic changes in the calcaneocuboidal joint (Table 2). Böhler's angle was restored in $70.1 \%$ (47 of 67 ) of the cases (Table 2). A total of $85.1 \%$ (57 of 67 ) cases had a reduction in calcaneal height to within $10 \%$ when compared to the non-operative side. Additionally, a reduction in the length of the calcaneus occurred within 10 in $94 \%$ (63 of 67) of cases when compared to the non-operative side. Thirty-eight $(56.8 \%)$ of the 67 patients had a widening of the calcaneus to within $10 \%$ compared to the opposite side.

\section{Complications}

Of the 92 surgically treated calcaneus fractures, $76(82.6 \%)$ healed without complications. In nine $(9.8 \%)$ cases, superficial skin infections, perforations of the Kirschner wires and bone dystrophy occurred and healed without any further complications. Significant complications occurred in six (6.5\%) cases: three cases had osteitis of the calcaneus, one case had dislocation of the fracture requiring revision surgery and two cases had peroneal tendon impingement. Three cases of osteitis healed through conservative therapy with oral antibiotics and the two patients with impingement of the peroneal tendon refused any operative intervention.

Using our method, disturbance of wound healing with skin and soft tissue necrosis requiring operative intervention was not observed. Additionally, no lower leg amputations and no total or partial calcanectomies had to be performed.

\section{Statistical analysis}

Using the Chi-square test a statistical significant correlation was found between the fracture type and the incidence of subtalar arthritis $(p<0.0268)$. Using the same test there was no statistical significant correlation found between the fracture type and quality of reduction $(p>0.6522)$, between fracture type and clinical result $(p>0.3204)$ and between fracture type and Böhler's angle $(p>0.5488)$. However, a statistical significant correlation was found between the clinical result and subtalar arthritis $(p<0.0013)$ and between the incidence of subtalar arthritis and quality of reduction when using the Fisher`s exact test $(p<0.0003)$.

\section{Discussion}

To correct deformations of the calcaneus, spare the soft tissue and lower the complication rate, indirect and less invasive reduction and fixation techniques to treat calcaneal fractures have been developed [7, 10, 12-14, 16, 19, 21, $22]$. Besides techniques with percutaneous reduction and internal K-wire fixation [7, 14, 16, 21] percutaneous reduction techniques with external fixation are described in the literature $[12,13,19,22]$. In our department we have combined and modified previously described closed reduction and internal fixation techniques $[14,27,28]$ to create a minimally invasive technique. This method used the lower joint surface of the talus as a guide for remodeling the posterior facet and reconstruction of the calcaneus.

A multiple of different evaluation scores makes a comparison of the clinical results with published results difficult [11, 17, 20, 30]. Using the Zwipp score, $61.3 \%$ of the cases (41 of 67) treated by our modified method had good or very good results regardless of fracture type. In 38.7\% (26 of 67) of cases, a satisfactory or bad result was achieved. After open reduction and internal fixation with a plate, Rammelt et al. [15] and Boack et al. [2] reported good and very good results in 65 and $66 \%$ of cases, respectively with the use of the \pm 200 Zwipp score. Boack et al. [2] presented satisfactory or bad clinical results in $34 \%$ of cases also using the Zwipp score.

Sanders et al. [18] showed that under application of a lateral approach followed by internal fixation with a plate, the worst clinical results occurred with type IV fractures. In this study the worst clinical results were achieved in the treatment of type IV fractures although within our cases no statistical significant correlation between fracture type and clinical result could be demonstrated (Table 2).

Patient satisfaction is an essential criterion for the successful treatment of calcaneus fractures. With a comparable follow-up period of 5.4 years, Thermann et al. [23] reported that $48.3 \%$ of patients viewed their treatment outcome as good or very good after open reduction of their calcaneus fractures, $37.3 \%$ of patients judged their treatment outcome 
as satisfactory and sufficient and $14.4 \%$ judged their outcome as insufficient. Our treatment method had comparable results with $44.8 \%$ of patients considering their treatment outcome very good or good, $41.8 \%$ as satisfactory and sufficient and $13.4 \%$ as insufficient.

At last follow-up evaluation, $64.2 \%$ of patients in this study were able to wear normal shoes while $28.4 \%$ required orthopaedic shoes. Comparably, in the study by Therman et al. [23], $68.7 \%$ of the patients wore normal shoes and $16.8 \%$ required orthopaedic shoes.

Overall, satisfactory mobility of the joints adjoining the calcaneus was achieved with our surgical technique. In approximately half of our patients, complete range of motion in the upper ankle joint was achieved and in the lower ankle joint, approximately $60 \%$ of patients had no or low movement restriction. Similar mobility in the upper and lower ankle joints has been reported in the literature after open reduction and osteosynthesis with a plate $[5,23]$. However, Buch [6] reported a worse range of motion in the upper and lower ankle joint after performing a percutaneus wire osteosynthesis in 100 calcaneal fractures with a varus or valgus deviation of the back foot occurring in half of the cases. In this study, malpositions of the back foot of more than $5^{\circ}$ were diagnosed in $6(9 \%)$ cases of the 67 cases. Clinically relevant changes in the axis of the front foot were not observed.

The radiological evaluation of the joints adjoining the calcaneus showed arthritic changes in the upper ankle joint in two of our cases. Half of our cases showed arthritic changes in the lower ankle joint and in approximately a fifth of our evaluated cases, arthritic changes were found in the calcaneocuboidal joint. In our study, $66.7 \%$ of type-II fractures and $98 \%$ of type-III/IV fractures showed arthritic changes in the lower ankle joint. These results are similar to those presented by Thermann et al. [23], who showed arthritic changes in the lower ankle joint in $65.2 \%$ of typeII fractures and in $81.7 \%$ of type III/IV fractures.

Regardless of fracture type, anatomical restoration of the joint surface or a joint deviation of up to $2 \mathrm{~mm}$ of the posterior facet was achieved postoperatively in $82.1 \%$ of cases. A total of $86.7 \%$ of type-II fractures and $72.8 \%$ of type-III/ IV fractures achieved anatomical restoration of the posterior facet. These results are comparable to those presented in the literature with open reduction and internal fixation with plates or screws [2, 17, 23].

In this study, the average length of the surgery was $61 \mathrm{~min}$. This is considerably less than the average length of surgeries utilizing open reduction and internal fixation of 139 and $168 \mathrm{~min}[2,20]$.

In $9.8 \%$ of cases, complications such as superficial skin infections, perforation of the Kirschner wires and bone dystrophy healed with conservative therapy or after removal of the Kirschner wires. This rather insignificant rate of complications is similar to those described in the literature after operative treatment of calcaneal fractures utilizing a minimally invasive technique $[7,16,21]$.

The rate of significant complications in this study was $6.5 \%$. In two cases, an impingement of the peroneal tendon occurred. One case had a dislocation of the fragments requiring revision surgery. Three cases had infections of the calcaneus and were related to a traumatic soft tissue injury that healed with conservative therapy. With open reduction, skin and soft tissue necrosis [2] with possible cutaneous flaps have been reported in scattered cases [5, 8, 9, 15, 17]. Folk et al. [9] demonstrated that in $25 \%$ of cases, wound complications required an additional surgery after open reduction of calcaneus fractures $84 \%$ of the time. Abidi et al. [1] reported wound healing disturbances in $33 \%$ of cases following open reduction and internal fixation of calcaneal fractures. There have also been reports in the literature of partial or total calcanectomies [15], including amputations, as a result of calcaneal osteitis after open reduction [9, 17]. Sanders et al. [17] considered a lower leg amputation if a patient suffered continual osteitis. In the current study no disturbances of wound healing, skin or soft tissue necrosis that required microsurgical intervention were observed.

In this study no statistical significant correlation could be found between fracture type and the ability to restore Böhler`s angle. However, regardless of fracture type Stulik et al. [21] showed similar results using a comparable minimally invasive technique while Thermann et al. [23] had worse results following open reduction with internal fixation.

In our study, there was a slight reduction in the height and length of the operated calcaneus when compared to the non-operated side. With our closed reduction and internal fixation technique, more than half of the cases had a widening of the calcaneus of more than $10 \%$ when compared to the opposite side, which is an unsatisfactory result. At the same time only two patients had an impingement of the peroneal tendon that the patients considered tolerable.

In summary, we presented a minimally invasive technique for the treatment of intraarticular, dislocated calcaneus fractures and were able to produce results comparable to open techniques with a lower rate of serious complications. In the majority of cases, an almost identical Böhler angle and geometry of the calcaneus was achieved when compared to the opposite side at the time of last follow-up. Simple removal of the Kirschner wires and shorter surgery time decrease patient stress and must be recognized as an advantage of this minimally invasive technique. Thus, we feel that our minimally invasive technique is a viable alternative for the treatment of intraarticular, dislocated calcaneal fractures. 
Open Access This article is distributed under the terms of the Creative Commons Attribution Noncommercial License which permits any noncommercial use, distribution, and reproduction in any medium, provided the original author(s) and source are credited.

\section{References}

1. Abidi NA, Dhawan S, Gruen GS, Vogt MT, Conti SF (1998) Wound-healing risk factors after open reduction and internal fixation of calcaneal fractures. Foot Ankle Int 19(12):856-861

2. Boack DH, Wichelhaus A, Mittlmeier T, Hoffmann R, Haas NP (1998) Therapy of dislocated calcaneus joint fracture with the AO calcaneus plate. Chirurg 69(11):1214-1223

3. Brodèn B (1949) Roentgen examination of the subtaloid joint in fractures of the calcaneus. Acta Radiol 31:85-88

4. Brunner U, Schweiberer L (1996) Injuries of the talus and calcaneus. Unfallchirurg 99(2):136-151

5. Brunner UH, Betz A, Halama R (1991) The surgical treatment of calcaneus fractures. Orthopade 20(1):55-66

6. Buch J (1980) Wire osteosynthesis of the calcaneal fracture. Aktuel Chir 15:285-296

7. Buch J, Blauensteiner W, Scherafati T, Vischer HM, Fischer W (1989) Conservative treatment of calcaneus fracture versus repositioning and percutaneous bore wire fixation. A comparison of 2 methods. Unfallchirurg 92(12):595-603

8. Cavadas PC, Landin L (2007) Management of soft-tissue complications of the lateral approach for calcaneal fractures. Plast Reconstr Surg 120(2):459-466, Discussion 467-469

9. Folk JW, Starr AJ, Early JS (1999) Early wound complications of operative treatment of calcaneus fractures: analysis of 190 fractures. J Orthop Trauma 13(5):369-372

10. Gavlik JM, Rammelt S, Zwipp H (2002) Percutaneous, arthroscopically-assisted osteosynthesis of calcaneus fractures. Arch Orthop Trauma Surg 122(8):424-428, Epub 2002 June 20

11. Kiotka HB, Alexander IJ, Adelaar RS, Nunley JA, Myerson MS, Sanders M (1994) Clinical rating systems for the ankle, hindfoot, midfood, hallux and lesser toes. Foot Ankle Int 15(7):349-353

12. Magnan B, Bortolazzi R, Marangon A, Marino M, Dall'Oca C, Bartolozzi P (2006) External fixation for displaced intra-articular fractures of the calcaneum. J Bone Joint Surg Br 88(11):14741479

13. McGarvey WC, Burris MW, Clanton TO, Melissinos EG (2006) Calcaneal fractures: indirect reduction and external fixation. Foot Ankle Int 27(7):494-499

14. Poigenfurst J, Buch J (1988) Treatment of severe fractures of the calcaneus by reposition and percutaneous wire fixation. Unfallchirurg 91(11):493-501
15. Rammelt S, Barthel S, Biewener A, Gavlik JM, Zwipp H (2003) Calcaneus fractures. Open reduction and internal fixation. Zentralbl Chir 128(6):517-528

16. Russe OJ, Russe FG, Buch J, Ziernhold G, Nones R (1979) Clinical and radiologic late results following internal wire osteosyntheses of the calcaneus. Follow-up studies from four Austrian hospitals for accidents. Hefte Unfallheilkd 134:195-199

17. Sanders R, Fortin P, DiPasquale T, Walling A (1993) Operative treatment in 120 displaced intraarticular calcaneal fractures. Clin Orthop 290:87-95

18. Sanders R (2000) Displaced intra-articular fractures of the calcaneus. J Bone Joint Surg Am 82(2):225-250

19. Schepers T, Schipper IB, Vogels LM, Ginai AZ, Mulder PG, Heetveld MJ, Patka P (2007) Percutaneous treatment of displaced intra-articular calcaneal fractures. J Orthop Sci 12(1):22-27, Epub 2007 January 31

20. Schuh A, Hausel M (2000) Difficulties in evaluating follow-up outcome in calcaneus fracture managed with plate osteosynthesis. Unfallchirurg 103(4):295-300

21. Stulik J, Stehlik J, Rysavy M, Wozniak A (2006) Minimally-invasive treatment of intra-articular fractures of the calcaneum. J Bone Joint Surg Br 88(12):1634-1641

22. Talarico LM, Vito GR, Zyryanov SY (2004) Management of displaced intraarticular calcaneal fractures by using external ring fixation, minimally invasive open reduction, and early weightbearing. J Foot Ankle Surg 43(1):43-50

23. Thermann H, Hufner T, Schratt HE, Albrecht K, Tscherne H (1999) Treatment of intraarticular calcaneal fractures in adults. A treatment algorithm. Unfallchirurg 102(3):152-166

24. Thordarson DB, Latteier M (2003) Open reduction and internal fixation of calcaneal fractures with a low profile titanium calcaneal perimeter plate. Foot Ankle Int 24(3):217-221

25. Tscherne H, Oestern HJ (1982) A new classification of soft-tissue damage in open and closed fractures. Unfallheilkunde 85(3):111115

26. Tscherne H, Zwipp H (1993) Calcaneal fracture. In: Tscherne H, Schatzker J (eds) Major fractures of the pilon, the talus and the calcaneus. Springer, Berlin, pp 153-174

27. Westhues H (1934) A new treatment method for the calcaneus fracture. Arch Orthop Unfallchir 35:121

28. Wondrak E (1959) Treatment of petrous bone fractures by internal fixation. Zentralbl Chir 84(7):260-266

29. Zwipp H, Tscherne H, Wulker N, Grote R (1989) Intra-articular fracture of the calcaneus. Classification, assessment and surgical procedures. Unfallchirurg 92(3):117-129

30. Zwipp H (1994) Chirurgie des Fußes. Springer, Wien, pp 100-130 